\title{
Classification of Dementia in EEG with a Two- Layered Feed Forward Artificial Neural Network
}

\author{
G. Anuradha \\ Department of ECE \\ BSA Crescent Institute of Science and Technology \\ Chennai, India \\ bhavanandhi@yahoo.com
}

\author{
D. Najumnissa Jamal \\ Department of EIE \\ BSA Crescent Institute of Science and Technology \\ Chennai, India \\ najumnissa.d@crescent.education
}

\begin{abstract}
Dementia has become a global public health issue. The current study is focused on diagnosing dementia with Electro Encephalography (EEG). The detection of the advancement of the disease is carried out by detecting the abnormal behavior in EEG measurements. Assessment and evaluation of EEG abnormalities is conducted for all the subjects in order to detect dementia. EEG feature analysis, namely dominant frequency, dominant frequency variability, and frequency prevalence, is done for abnormal and normal subjects and the results are compared. For dementia with Lewy bodies, in $85 \%$ of the epochs, the dominant frequency is present in the delta range whereas for normal subjects it lies in the alpha range. The dominant frequency variability in $75 \%$ of the epochs is above $4 \mathrm{~Hz}$ for dementia with Lewy bodies, and in normal subjects at $72 \%$ of the epochs, the dominant frequency variability is less than $2 \mathrm{~Hz}$. It is observed that these features are sufficient to diagnose dementia with Lewy bodies. The classification of Lewy body dementia is done by using a feed-forward artificial neural network which proved to have a $\mathbf{9 4 . 4 \%}$ classification accuracy. The classification with the proposed feed-forward neural network has better accuracy, sensitivity, and specificity than the already known methods.
\end{abstract}

Keywords-Lewy body dementia; EEG; dementia; neural network; dominant frequency

\section{INTRODUCTION}

The term dementia refers to a syndrome that causes a decline in cognitive functions, mainly the person's memory and intelligence, due to the death of brain cells. Different types of dementia have their own characteristic features. In Alzheimer's disease, the pathology involves the reduction of neurons which help in stimulating cortex and hippocampus regions, deposits in blood vessels, and formation of amyloid plaques [1]. An intermediate stage called mild cognitive impairment was found between the normal cognitive and dementia. Subjects in this stage are not demented but exhibit a decline in memory beyond that expected at their age and education [2,3]. Mild cognitive impairment is an earlier stage, which may progress to dementia [4]. Psychometric predictors and clinical evaluation in elderly subjects can predict mild cognitive impairment [5]. Mild cognitive impairment and dementia can be detected using EEG biomarkers [6-8]. Mini Mental State Examination (MMSE), Clinical Dementia Rating (CDR), and Global Deterioration
Scale (GDS) are the subjective measures used to evaluate the early stages of mild dementia $[3,4,11]$.

Alzheimer's Disease (AD) is the most common form of dementia $[9,10]$. In AD patients, the decrease in consistency of fast rhythms and shift of power spectrum to lower frequencies, are the abnormalities in EEG signals [13]. The second most common dementia is Vascular Dementia ( $\mathrm{VaD})$ [14]. $\mathrm{VaD}$ is caused by the ischemic brain lesions due to cardiovascular pathology resulting in decline of the cognitive functions [1517]. Another type of dementia, Lewy Body Dementia (LBD) is characterized by Lewy bodies with large old plaques. Fronto Temporal Dementia (FTD) might occur in middle aged people and is characterized by the degeneration of prefrontal and anterior temporal cortex associated with personality changes and changes in social conduct [18]. Parkinsonism consists of problems in motor nerves namely, resting tremor, rigidity and freezing [19].

Finding a biomarker to detect dementia at an earlier stage is a major challenge. The biomarker should detect the neuropathological processes caused by dementia to help the effective treatment of the disease [10]. EEG can function as such a biomarker to identify those physiological and pathological conditions. EEG in addition to functioning as a diagnosis tool also differentiates the stages of dementia [20]. The reduced cost and non-invasiveness of the EEG electrodes makes it suitable for detecting dementia and its progression $[10,11]$. The challenges in an EEG biomarker are the accurate and early detection of the disease [6]. The identification of different stages of dementia and the precise detection is made possible by EEG [21]. Slowing of EEG dominant posterior rhythm frequency and reduced alpha and beta activities are observed in visual characteristics of AD. Reductions in mean frequency and alpha and beta power are observed in the analysis of EEG signals of AD patients, in earlier stages of dementia [4]. Mild dementia is characterized by an increase in theta activity and severe dementia by a decrease in beta activity $[22,23]$. Resting state eyes-closed EEG recording is used to assess the progression from mild to severe AD. The stage of mild cognitive impairment is characterized by increase in delta power and decrease in alpha and beta power [24]. Early stages of LBD show reduction in cognitive functions like attention, problem solving, and visuospatial skills. Changes in cognitive 
function and Parkinsonism are the characteristic features that differentiate DLB from AD and other dementias [25]. LBD and $\mathrm{AD}$ are distinguished in early stages by quantitative analysis on the EEG signal. The dominant frequency of $\mathrm{AD}$ patients were in alpha range, in greater than $60 \%$ of the epochs and dominant frequency variability in $\mathrm{AD}$ was less than $1.6 \mathrm{~Hz}$ whereas $\mathrm{LBD}$ patients had dominant frequency variability greater than $4 \mathrm{~Hz}$ [26-28].

In our present study, classification of LBD and control (normal) subjects is achieved with the help of a feed forward neural network with features extracted in the frequency domain [33]. This paper aims in finding whether these abnormalities are present in the EEG and whether these can help in the detection and classification of dementia to improve diagnostic accuracy.

\section{MATERIALS AND METHODS}

The proposed method consists of EEG acquisition, preprocessing, feature extraction, and classification as shown in Figure 1. EEG recording was done with subjects awake, relaxed, and with eyes closed. The sampling rate was $173.6 \mathrm{~Hz}$. Two-second long epochs were chosen for analysis [28, 33]. A total of 12 abnormal and 18 normal EEG records were obtained. Fast Fourier Transform (FFT), $M(K)$ was performed for the EEG time series $m(n)$. Spectral amplitude $Y_{j}$ was evaluated for each epoch, with $j$ being the number of epochs. The frequency at which $Y_{j}$ is maximum, among all epochs is the dominant frequency value $F_{j}$. The Mean Dominant Frequency (MDF) of all epochs was found. Dominant Frequency Range (DFR), the range of maximum and minimum value of $F_{j}$, Dominant Frequency Variability (DFV), the variation of the dominant frequency, i.e. the difference between the maximum and minimum value of $F_{j}$, Frequency Prevalence (FP) which indicates the existence of a frequency $F_{j}$ in alpha, theta, or delta range were evaluated. The above features are extracted from the preprocessed signal which is applied for classification to the feed forward neural network as normal and demented signals. In the proposed method, Levenberg algorithm is used to update the weights of the feed forward neural network.

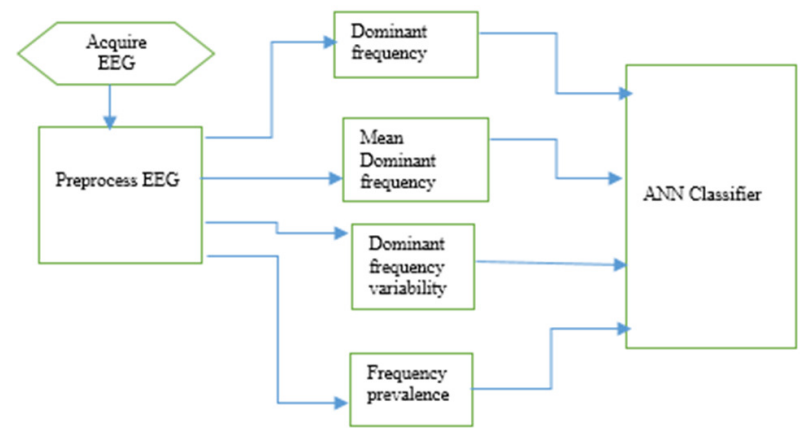

Fig. 1. Flow chart of the proposed method.

Machine learning algorithms learn from the past experience and provide optimized performance criteria. The most important aspect of a learning algorithm is its accuracy.
Statistical learning techniques are developed and compared using pattern recognition which is one of the key aspects of artificial intelligence. Linear Discriminant Analysis (LDA) and neural networks, Support Vector Machine (SVM) [29, 30], Linear Support Vector Machine (LSVM), Balanced Communication-Avoiding Support Vector Machine (BCASVM) [32], and Levenberga algorithm [33] are some of the available classifiers.

In the proposed method, accuracy, i.e. the differentiation of subjects with LBD from normal subjects, sensitivity which determines LBD correctly, and specificity which determines the healthy subjects correctly, were obtained with a twolayered feed forward neural network. Simulation of the above study is done using the EEG data obtained from the Bonn data base [32].

\section{RESULTS AND DISCUSSION}

Analysis of Dominant Frequency (DF), MDF, DFR, DFV, and FP in both normal and abnormal EEGs was conducted. Table I shows the features extracted from 12 abnormal EEGs. For every subject, the maximum DF among all epochs was chosen. It is observed that, the DF in almost all subjects, except subject 9, falls in the delta range. MDF is present in the delta range in all the analyzed subjects. The DFRs of subjects 2,4 , and 8 fall completely in the delta range. The DFV of $50 \%$ of the subjects is present in the delta and theta range. Above $80 \%$ of the subjects have FP in the delta range. From the feature analysis of abnormal subjects, it is found that, the MDF of all the epochs is found to be greater than $0.9 \mathrm{~Hz}$ and the DFV is greater than $4 \mathrm{~Hz}$ in $75 \%$ of epochs which confirms LBD similar to pattern 4 [27].

TABLE I. FEATURES OF LDB

\begin{tabular}{|c|c|c|c|c|c|}
\hline S.NO & DF (Hz) & MDF (Hz) & DFR (Hz) & DFV (Hz) & FP (\%) \\
\hline 1 & 1.3 & 1.0 & $0-4.4$ & 4.0 & $\mathrm{D}=90 \mathrm{~T}=10$ \\
\hline 2 & 3.3 & 0.9 & $0-3.3$ & 3.0 & $\mathrm{D}=100$ \\
\hline 3 & 0.6 & 1.6 & $0-11.5$ & 12.0 & $\mathrm{D}=90 \mathrm{~A}=10$ \\
\hline 4 & 0.6 & 1.0 & $0.3-2.0$ & 2.0 & $\mathrm{D}=100$ \\
\hline 5 & 1.3 & 1.6 & $0-5-7.0$ & 6.0 & $\mathrm{D}=80 \mathrm{~T}=20$ \\
\hline 6 & 0.3 & 1.4 & $0.0-11.8$ & 12.0 & $\mathrm{D}=80 \mathrm{~A}=20$ \\
\hline 7 & 0.3 & 3.5 & $0.3-11.1$ & 11.0 & $\mathrm{D}=73 \mathrm{~A}=27$ \\
\hline 8 & 1.0 & 1.29 & $0.3-3.0$ & 3.0 & $\mathrm{D}=100$ \\
\hline 9 & 10.8 & 2.9 & $0.0-11.8$ & 12.0 & $\mathrm{D}=80 \mathrm{~A}=20$ \\
\hline 10 & 0.6 & 2.2 & $0.3-11.5$ & 12.0 & $\mathrm{D}=90 \mathrm{~A}=10$ \\
\hline 11 & 0.339 & 4.0 & $0.0-11.5$ & 11.5 & $\mathrm{D}=58 \mathrm{~A}=42$ \\
\hline 12 & 0.339 & 4.2 & $0.33-12.2$ & 11.86 & $\mathrm{D}=67 \mathrm{~A}=33$ \\
\hline \multicolumn{7}{|c}{$\mathrm{D}$ delta 1-3.9Hz, T: theta 4-5.5Hz, A: alpha 8-12Hz }
\end{tabular}

Table II shows the features extracted from normal EEGs. It is observed that, the DF in more than the $80 \%$ subjects falls in the alpha range. MDF is present in alpha range $80 \%$ of the analyzed subjects. The DF range of $83 \%$ of the subjects falls in the delta range. The DFV of $80 \%$ of the subjects is present in the delta range. Above $70 \%$ of the subjects have FP in the alpha range. Figure 2 shows the comparison of the DF for LBD and normal subjects. The DF for LBD is present in the $85 \%$ of the epochs in delta range, between $0.3 \mathrm{~Hz}$ and $3.3 \mathrm{~Hz}$. The DF of subject 9 alone falls in the alpha $(10.8 \mathrm{~Hz})$ in LBD, whereas subjects, except 3,15 , and 16 , exhibit DF in alpha, between $10 \mathrm{~Hz}$ and $11 \mathrm{~Hz}$. The $85 \%$ of the epochs in normal subjects have DF in the alpha range. 
TABLE II. FEATURES OF NORMAL SUBJECTS

\begin{tabular}{|c|c|c|c|c|c|}
\hline S.NO & DF (Hz) & MDF (Hz) & DFR(Hz) & DFV(Hz) & FP (\%) \\
\hline 1 & 10.5 & 3.2 & $0.0-10.5$ & 10.5 & $\mathrm{D}=75 \mathrm{~A}=25$ \\
\hline 2 & 11.0 & 11.0 & $11.0-12.0$ & 1.0 & $\mathrm{~A}=100$ \\
\hline 3 & 14.0 & 14.0 & $12.0-14.0$ & 2.0 & $\mathrm{~A}=100$ \\
\hline 4 & 11.0 & 11.5 & $11.0-12.0$ & 1.0 & $\mathrm{D}=25 \mathrm{~A}=75$ \\
\hline 5 & 11.0 & 11.5 & $11.0-12.0$ & 1.0 & $\mathrm{D}=100$ \\
\hline 6 & 10.8 & 11.0 & $10.5-11.0$ & 1.02 & $\mathrm{~A}=100$ \\
\hline 7 & 11.0 & 11.29 & $11.0-12.0$ & 1.0 & $\mathrm{~A}=100$ \\
\hline 8 & 11.0 & 11.25 & $11.0-12.0$ & 1.0 & $\mathrm{D}=75 \mathrm{~A}=25$ \\
\hline 9 & 11.0 & 11.13 & $11.0-12.0$ & 1.0 & $\mathrm{~A}=100$ \\
\hline 10 & 11.0 & 11.55 & $11.0-12.0$ & 1.0 & $\mathrm{~A}=100$ \\
\hline 11 & 11.0 & 11.61 & $11.0-12.0$ & 1.0 & $\mathrm{~A}=100$ \\
\hline 12 & 11.0 & 11.5 & $11.0-12.0$ & 1.0 & $\mathrm{D}=8 \mathrm{~A}=92$ \\
\hline 13 & 11 & 10 & $9-11$ & 2 & $\mathrm{D}=8 \mathrm{~A}=92$ \\
\hline 14 & 11 & 11 & $11-12$ & 1 & $\mathrm{D}=25 \mathrm{~A}=75$ \\
\hline 15 & 15 & 13 & $10-16$ & 6 & $\mathrm{~A}=100$ \\
\hline 16 & 14 & 14 & $8-15$ & 7 & $\mathrm{~A}=100$ \\
\hline 17 & 11 & 11.5 & $11-12$ & 1 & $\mathrm{~A}=100$ \\
\hline 18 & 11 & 11.5 & $11-12$ & 1 & $\mathrm{~A}=100$ \\
\hline
\end{tabular}

D: delta $1-3.9 \mathrm{~Hz}, \mathrm{~T}$ : theta $4-5.5 \mathrm{~Hz}, \mathrm{~A}$ : alpha $8-12 \mathrm{~Hz}$

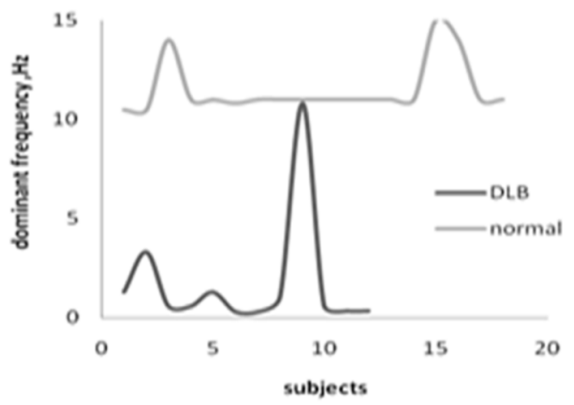

Fig. 2. DF of LBD and normal subjects.

The calculated MDF for LBD and normal subjects is shown in Figure 3. All the normal subjects, except 1, 3, 15, and 16, have MDF in alpha, between $11 \mathrm{~Hz}$ and $12 \mathrm{~Hz}$, whereas in LBD, the MDF falls in the delta range of $0.9 \mathrm{~Hz}$ and $3.5 \mathrm{~Hz}$ in subjects 1 to 10 and theta range of $4 \mathrm{~Hz}$ and $4.2 \mathrm{~Hz}$ in subjects 11 and 12 respectively.

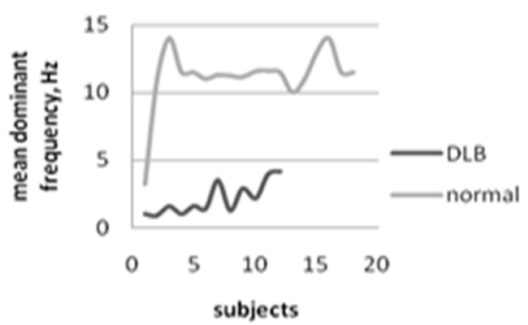

Fig. 3. MDF of LBD and normal subjects.

Figure 4 shows the comparison of DFV for subjects with LBD and normal subjects. In $75 \%$ of the epochs, DFV above $4 \mathrm{~Hz}$ is observed in dementia and in normal subjects $83 \%$ of the epochs have DFV less than 2Hz. All LBD subjects, except 2, 4, and 8 , have DFV above $4 \mathrm{~Hz}$. All normal subjects, except 1,15 , and 16, have DFV less than $2 \mathrm{~Hz}$. The calculated FP for LBD and normal subjects is shown in Figure 5 . More than $80 \%$ of the epochs in LBD have FP in the delta range [24], whereas for normal subjects $80 \%$ of the epochs are in the alpha range. It is therefore found that EEG abnormalities can function as a marker for the detection of LBD.

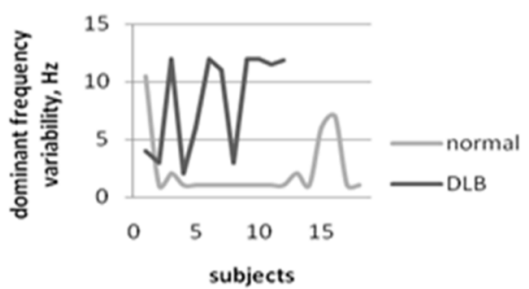

Fig. 4. DFV for LBD and normal subjects.

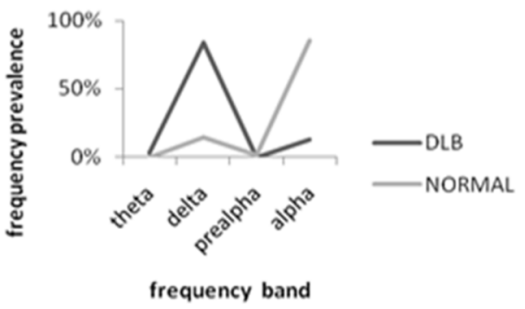

Fig. 5. FP for LBD and normal subjects.

\section{ClassificATION}

The extracted features DF, MDF, and DFV were utilized in a feed forward neural network implemented in MatLab. Levenberg algorithm was used to update the weights of the neural network. Accuracy, sensitivity, and specificity were used to evaluate the classification performance. These parameters are calculated as:

$$
\begin{gathered}
\text { Accuracy }=\frac{\mathrm{TP}+\mathrm{TN}}{\mathrm{TP}+\mathrm{TN}+\mathrm{FP}+\mathrm{FN}} \\
\text { Sensitivity }=\frac{\mathrm{TP}}{\mathrm{TP}+\mathrm{FN}} \\
\text { Specificity }=\frac{\mathrm{TN}}{\mathrm{TN}+\mathrm{FP}}
\end{gathered}
$$

where TP, TN, FP, FN stand for the true positive, true negative, false positive and false negative classifications respectively.

The confusion matrix evaluates the overall performance of a classification method. Accuracy, sensitivity, and specificity of each feature are obtained from the confusion matrix. Figure 6 shows the confusion matrix obtained for classification using the feature DFV. Accuracy of $88.9 \%$, sensitivity of $83.3 \%$, and specificity of $100 \%$ were achieved. Figure 7 shows the confusion matrix obtained for classification using DF, with accuracy of $94.4 \%$, sensitivity of $90.9 \%$, and specificity of $100 \%$ achieved. Figure 8 shows the confusion matrix obtained for MDF. The acquired accuracy, sensitivity, and specificity were $94.4 \%, 88.9 \%$ and $100 \%$ respectively.

Table III shows the accuracy, sensitivity, and specificity using the classifiers spectral SVM, decision tree with Fourier coefficients, and SVM with spectral peak [30, 35, 36]. The classifier performance is poor in Fourier coefficients with decision tree, where the accuracy, sensitivity and specificity are less than $75 \%$, whereas spectral svm shows good classifier performance, with accuracy of $86 \%$, sensitivity of $88 \%$ and 
specificity of $84 \%$. The accuracy, sensitivity, and specificity of the proposed feed forward neural network classifier with features like MDF, DFV and DF obtained from the confusion matrix is shown in Table IV.

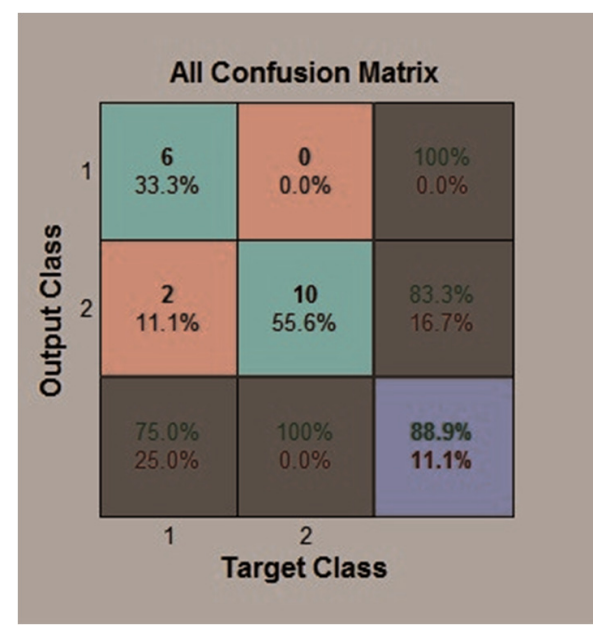

Fig. 6. Confusion matrix for DFV.

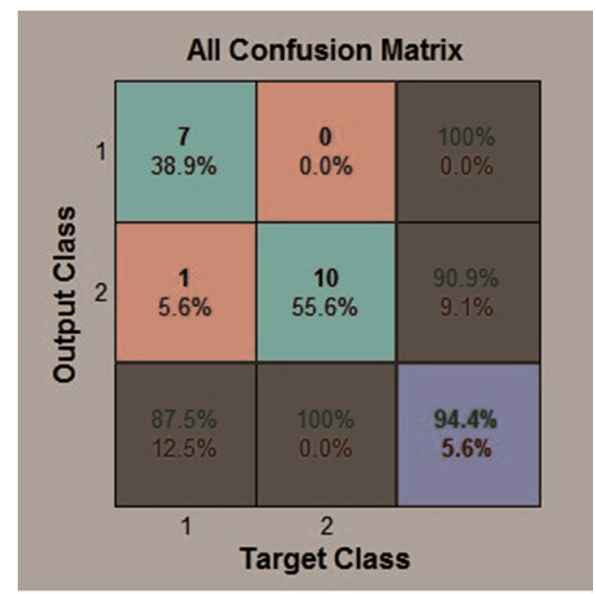

Fig. 7. Confusion matrix for DF.

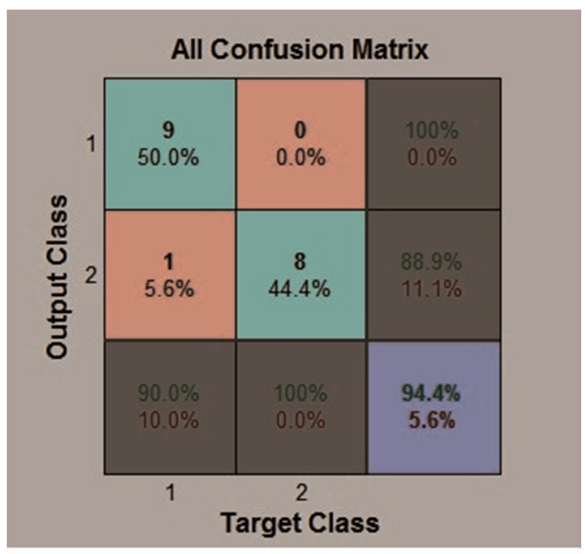

Fig. 8. Confusion matrix for MDF.
TABLE III. ACCURACY, SENSITIVITY, AND SPECIFICITY OF OTHER CLASSIFIERS

\begin{tabular}{|c|c|c|c|}
\hline Feature & $\begin{array}{c}\text { Accuracy } \\
(\mathbf{\%})\end{array}$ & $\begin{array}{c}\text { Sensitivity } \\
(\mathbf{\%})\end{array}$ & $\begin{array}{c}\text { Specificity } \\
(\mathbf{\%})\end{array}$ \\
\hline Spectral SVM & 86 & 88 & 84 \\
\hline $\begin{array}{c}\text { Fourier coefficients with } \\
\text { decision tree }\end{array}$ & 72.2 & 72.2 & 59.4 \\
\hline Spectral peak SVM & 79.9 & 83.2 & 76.4 \\
\hline
\end{tabular}

TABLE IV. ACCURACY, SENSITIVITY, AND SPECIFICITY OF THE NN CLASSIFIER

\begin{tabular}{|c|c|c|c|}
\hline Feature & $\begin{array}{c}\text { Accuracy } \\
(\%)\end{array}$ & $\begin{array}{c}\text { Sensitivity } \\
(\%)\end{array}$ & $\begin{array}{c}\text { Specificity } \\
(\%)\end{array}$ \\
\hline NN with MDF & 94.4 & 88.9 & 100 \\
\hline NN with DFV & 88.9 & 83.3 & 100 \\
\hline NN with DF & 94.4 & 90.9 & 100 \\
\hline
\end{tabular}

The highest percentage of sensitivity of the proposed classifier is $90.9 \%$ with the DF feature. The specificity obtained for the proposed classifier is $100 \%$ for MDF, DFV, and DF, while the accuracy is $94.4 \%$ in both MDF and DF. Hence, DF produces better results when compared to the other features, MDF and DFV. This type of classification method with a two-layered feed forward $\mathrm{NN}$ using dominant frequency feature gives better sensitivity, specificity, and accuracy when compared with other existing methods.

\section{CONCLUSION}

In this study, EEG analysis was performed on both abnormal and healthy subjects. Dominant frequency analysis was proposed to extract spectral features from EEG signals. The spectral features obtained are sufficient for the diagnosis of LBD [30]. An artificial neural network was used for classification. The experimental results show that the proposed two-layered feed forward artificial neural network-based classifier can achieve better classification performance than the existing LBD diagnosis methods.

\section{REFERENCES}

[1] J. Wu, Y. Yu, and J. Yang, "Neuropsychological Parameters as Potential Biomarkers for Alzheimer's Disease," Current Translational Geriatrics and Experimental Gerontology Reports, vol. 1, no. 2, pp. 68-75, Jun. 2012, https://doi.org/10.1007/s13670-012-0007-4.

[2] S. Fahn, "Description of Parkinson's disease as a clinical syndrome," Annals of the New York Academy of Sciences, vol. 991, pp. 1-14, Jun. 2003, https://doi.org/10.1111/j.1749-6632.2003.tb07458.x.

[3] P. M. Rossini, S. Rossi, C. Babiloni, and J. Polich, "Clinical neurophysiology of aging brain: From normal aging to neurodegeneration," Progress in Neurobiology, vol. 83, no. 6, pp. 375 400, Dec. 2007, https://doi.org/10.1016/j.pneurobio.2007.07.010.

[4] R. C. Petersen et al., "Apolipoprotein E Status as a Predictor of the Development of Alzheimer's Disease in Memory-Impaired Individuals," Journal of the American Medical Informatics Association, vol. 273, no. 16, pp. 1274-1278, Apr. 1995, https://doi.org/10.1001/jama.1995. 03520400044042 .

[5] C. Flicker, S. H. Ferris, and B. Reisberg, "Mild cognitive impairment in the elderly: Predictors of dementia," Neurology, vol. 41, no. 7, pp. 10061006, Jul. 1991, https://doi.org/10.1212/WNL.41.7.1006.

[6] J. Jeong, "EEG dynamics in patients with Alzheimer's disease," Clinical Neurophysiology, vol. 115, no. 7, pp. 1490-1505, Jul. 2004, https://doi.org/10.1016/j.clinph.2004.01.001.

[7] G. Henderson et al., "Development and assessment of methods for detecting dementia using the human electroencephalogram," IEEE 
Transactions on Biomedical Engineering, vol. 53, no. 8, pp. 1557-1568, Aug. 2006, https://doi.org/10.1109/TBME.2006.878067.

[8] Y. E. Geda, "Mild Cognitive Impairment in Older Adults," Current Psychiatry Reports, vol. 14, no. 4, pp. 320-327, Aug. 2012, https://doi.org/10.1007/s1 1920-012-0291-x.

[9] M. P. Mattson, "Pathways towards and away from Alzheimer's disease," Nature, vol. 430, no. 7000, pp. 631-639, Aug. 2004, https://doi.org/ 10.1038 /nature 02621 .

[10] A. Cedazo-Minguez and B. Winblad, "Biomarkers for Alzheimer's disease and other forms of dementia: Clinical needs, limitations and future aspects," Experimental Gerontology, vol. 45, no. 1, pp. 5-14, Jan. 2010, https://doi.org/10.1016/j.exger.2009.09.008.

[11] B. Reisberg, S. H. Ferris, M. J. de Leon, and T. Crook, "Global Deterioration Scale (GDS)," Psychopharmacology Bulletin, vol. 24, no. 4, pp. 661-663, 1988.

[12] R. C. Petersen, G. E. Smith, S. C. Waring, R. J. Ivnik, E. G. Tangalos, and E. Kokmen, "Mild Cognitive Impairment: Clinical Characterization and Outcome," Archives of Neurology, vol. 56, no. 3, pp. 303-308, Mar. 1999, https://doi.org/10.1001/archneur.56.3.303.

[13] P. Luu, D. M. Tucker, R. Englander, A. Lockfeld, H. Lutsep, and B. Oken, "Localizing Acute Stroke-related EEG Changes: Assessing the Effects of Spatial Undersampling," Journal of Clinical Neurophysiology, vol. 18, no. 4, pp. 302-317, Jul. 2001.

[14] P. D. Meek, E. K. McKeithan, and G. T. Schumock, "Economic Considerations in Alzheimer's Disease," Pharmacotherapy: The Journal of Human Pharmacology and Drug Therapy, vol. 18, no. 2P2, pp. 68 73, 1998, https://doi.org/10.1002/j.1875-9114.1998.tb03880.x.

[15] J. T. Coyle, D. L. Price, and M. R. DeLong, "Alzheimer's disease: a disorder of cortical cholinergic innervation," Science, vol. 219, no. 4589, pp. 1184-1190, Mar. 1983, https://doi.org/10.1126/science.6338589.

[16] A. V. Terry and J. J. Buccafusco, "The Cholinergic Hypothesis of Age and Alzheimer's Disease-Related Cognitive Deficits: Recent Challenges and Their Implications for Novel Drug Development," Journal of Pharmacology and Experimental Therapeutics, vol. 306, no. 3, pp. 821827, Sep. 2003, https://doi.org/10.1124/jpet.102.041616.

[17] C. McVeigh and P. Passmore, "Vascular dementia: prevention and treatment," Clinical Interventions in Aging, vol. 1, no. 3, pp. 229-235, Sep. 2006.

[18] G. C. Roman, "Vascular Dementia: Distinguishing Characteristics, Treatment, and Prevention," Journal of the American Geriatrics Society, vol. 51, no. 5s2, pp. S296-S304, 2003, https://doi.org/10.1046/j.15325415.5155.x.

[19] D. R. Thal, L. T. Grinberg, and J. Attems, "Vascular dementia: Different forms of vessel disorders contribute to the development of dementia in the elderly brain," Experimental Gerontology, vol. 47, no. 11, pp. 816824, Nov. 2012, https://doi.org/10.1016/j.exger.2012.05.023.

[20] H. Hampel et al., "Perspective on future role of biological markers in clinical therapy trials of Alzheimer's disease: A long-range point of view beyond 2020," Biochemical Pharmacology, vol. 88, no. 4, pp. 426-449, Apr. 2014, https://doi.org/10.1016/j.bcp.2013.11.009.

[21] U. P. Mosimann and I. G. McKeith, "Dementia with lewy bodies-diagnosis and treatment," Swiss medical weekly, vol. 133, no. 9-10, pp. 131-142, Mar. 2003, https://doi.org/10.7892/boris.43041.

[22] L. A. Coben, W. L. Danziger, and L. Berg, "Frequency analysis of the resting awake EEG in mild senile dementia of Alzheimer type," Electroencephalography and Clinical Neurophysiology, vol. 55, no. 4, pp. 372-380, Apr. 1983, https://doi.org/10.1016/0013-4694(83)90124-4.

[23] L. A. Coben, W. Danziger, and M. Storandt, "A longitudinal EEG study of mild senile dementia of Alzheimer type: changes at 1 year and at 2.5 years," Electroencephalography and Clinical Neurophysiology, vol. 61, no. 2, pp. 101-112, Aug. 1985, https://doi.org/10.1016/00134694(85)91048-X.

[24] C. Babiloni et al., "Cortical sources of resting state electroencephalographic alpha rhythms deteriorate across time in subjects with amnesic mild cognitive impairment," Neurobiology of Aging, vol. 35, no. 1, pp. 130-142, Jan. 2014, https://doi.org/ 10.1016/j.neurobiolaging.2013.06.019.
[25] I. G. McKeith et al., "Consensus guidelines for the clinical and pathologic diagnosis of dementia with Lewy bodies (DLB): Report of the consortium on DLB international workshop," Neurology, vol. 47, no. 5, pp. 1113-1124, Nov. 1996, https://doi.org/10.1212/WNL.47.5.1113.

[26] L. Bonanni, A. Thomas, P. Tiraboschi, B. Perfetti, S. Varanese, and M. Onofrj, "EEG comparisons in early Alzheimer's disease, dementia with Lewy bodies and Parkinson's disease with dementia patients with a 2year follow-up," Brain, vol. 131, no. 3, pp. 690-705, Mar. 2008, https://doi.org/10.1093/brain/awm322.

[27] S.-C. Du, D.-L. Huang, and H. Wang, "An Adaptive Support Vector Machine-Based Workpiece Surface Classification System Using HighDefinition Metrology," IEEE Transactions on Instrumentation and Measurement, vol. 64, no. 10, pp. 2590-2604, Oct. 2015, https://doi.org/ 10.1109/TIM.2015.2418684.

[28] N. N. Kulkarni and V. K. Bairagi, "Extracting Salient Features for EEGbased Diagnosis of Alzheimer's Disease Using Support Vector Machine Classifier," IETE Journal of Research, vol. 63, no. 1, pp. 11-22, Jan. 2017, https://doi.org/10.1080/03772063.2016.1241164.

[29] S. Du, C. Liu, and L. Xi, "A Selective Multiclass Support Vector Machine Ensemble Classifier for Engineering Surface Classification Using High Definition Metrology," Journal of Manufacturing Science and Engineering, vol. 137, Feb. 2015, Art. no. 011003, https://doi.org/ $10.1115 / 1.4028165$.

[30] L. Bonanni et al., "Quantitative electroencephalogram utility in predicting conversion of mild cognitive impairment to dementia with Lewy bodies," Neurobiology of Aging, vol. 36, no. 1, pp. 434-445, Jan. 2015, https://doi.org/10.1016/j.neurobiolaging.2014.07.009.

[31] R. G. Andrzejak, K. Lehnertz, F. Mormann, C. Rieke, P. David, and C. E. Elger, "Indications of nonlinear deterministic and finite-dimensional structures in time series of brain electrical activity: Dependence on recording region and brain state," Physical Review E, vol. 64, no. 6, Nov. 2001, Art. no. 061907, https://doi.org/10.1103/PhysRevE.64. 061907.

[32] "EEG time series download page," Department of Epileptology, University of Bonn. http://epileptologie-bonn.de/cms/upload/workgroup/ lehnertz/eegdata.html (accessed Mar. 24, 2021).

[33] G. Anuradha, N. Jamal, and S. Rafiammal, "Detection of dementia in EEG signal using dominant frequency analysis," in IEEE International Conference on Power, Control, Signals and Instrumentation Engineering, Chennai, India, Sep. 2017, pp. 710-714, https://doi.org/ 10.1109/ICPCSI.2017.8391806.

[34] M. T. Hagan and M. B. Menhaj, "Training feedforward networks with the Marquardt algorithm," IEEE Transactions on Neural Networks, vol. 5, no. 6, pp. 989-993, Nov. 1994, https://doi.org/10.1109/72.329697.

[35] G. Fiscon et al., "Combining EEG signal processing with supervised methods for Alzheimer's patients classification," BMC Medical Informatics and Decision Making, vol. 18, no. 1, May 2018, Art. no. 35, https://doi.org/10.1186/s12911-018-0613-y.

[36] L. R. Trambaiolli, A. C. Lorena, F. J. Fraga, P. A. M. Kanda, R. Anghinah, and R. Nitrini, "Improving Alzheimer's Disease Diagnosis with Machine Learning Techniques," Clinical EEG and Neuroscience, vol. 42, no. 3, pp. 160-165, Jul. 2011, https://doi.org/10.1177/ 155005941104200304.

[37] S. S. Rafiammal, D. N. Jamal, and S. K. Mohideen, "Reconfigurable Hardware Design for Automatic Epilepsy Seizure Detection using EEG Signals," Engineering, Technology \& Applied Science Research, vol. 10, no. 3, pp. 5803-5807, Jun. 2020, https://doi.org/10.48084/etasr.3419.

[38] M. B. Ayed, "Balanced Communication-Avoiding Support Vector Machine when Detecting Epilepsy based on EEG Signals," Engineering, Technology \& Applied Science Research, vol. 10, no. 6, pp. 6462-6468, Dec. 2020, https://doi.org/10.48084/etasr.3878. 